\title{
ACHADOS CIRÚRGICOS EM 260 CASOS DE IMPRESSÃO BASILAR E/OU MALFORMAÇÃO DE ARNOLD-CHIARI
}

\author{
JOSÉ ALBERTO GONÇALVES DA SILVA*, JOSÉ CORREIA DE FARIAS BRITO**, \\ PAULO VIRGOLINO DA NÓBREGA**, MARIA DO DESTERRO LEIROS COSTA **, \\ ALIANE BARBOSA LEAL DE SOUZA **
}

\begin{abstract}
RESUMO - A impressão basilar é malformação frequentemente observada no Nordeste do Brasil. No período de 1971 a 1992 foram operados, em nosso Serviço, 260 pacientes com malformações occipitocervicais, sendo $29(11,1 \%)$ casos de impressão basilar pura, $18(6,9 \%)$ com malformação de Arnold-Chiari e $213(81,9 \%)$ com impressão basilar associada à malformação de Arnold-Chiari. São relatados os achados cirúrgicos do plano ósseo, da dura-máter, do tecido nervoso e dos vasos da fossa posterior.
\end{abstract}

PALAVRAS-CHAVE: impressão basilar, malformação de Arnold-Chiari, anormalidade craniovertebral, achados cirúrgicos.

\section{Basilar impression and Arnold-Chiari malformation: surgical findings in $\mathbf{2 6 0}$ cases}

SUMMARY - The surgical findings observed in a series of 260 patients with craniovertebral anomaly are presented. There were $29(11.1 \%)$ patients only with basilar impression, $18(6.9 \%)$ cases of pure Arnold-Chiari malformation and $213(81.9 \%)$ cases of basilar impression associated with Chiari malformation. The surgical findings on bone, dura-mater, nervous tissue and blood vessels of this region are reported. findings.

KEY WORDS: basilar impression, Arnold-Chiari malformation, craniovertebral abnormality, surgical

A impressão basilar ocupa lugar de destaque entre as malformações occipitocervicais. A forma primária decorre de erro embriogênico, enquanto a forma secundária é causada por afecções ósseas diversas, como doença de Paget, displasia fibrosa, osteoporose e osteomalácia. A descrição original da impressão basilar foi feita por Ackermann ${ }^{1}$, em 1790, em estudos sobre o cretinismo. Virchow ${ }^{32}$, em 1857, denominou-a de platibasia e rejeitou a relação entre impressão basilar e cretinismo, como fora admitida por Ackermann ${ }^{1}$. Virchow ${ }^{33}$, em 1876, denominou a malformação de impressão basilar, tendo descrito detalhadamente as modificações ósseas e sendo o primeiro autor a admitir a origem congênita em muitos de seus casos. Homén ${ }^{23}$, em 1901, foi o primeiro autor a correlacionar os dados clínicos com os achados anátomo-patológicos. A necropsia de seu paciente evidenciou achatamento do bulbo e cerebelo, bem como presença de cavidade cística na medula espinhal. Explicou a patogenia dos sintomas clínicos através da compressão das estruturas comprometidas na invaginação da apófise odontóide. Quanto à clínica, a impressão basilar sintomática pode apresentar síndromes clínicas diversas, isoladas ou combinadas, tais como síndrome dos nervos intracranianos, síndromes sensitivas superficial e profunda, cerebelar, de hipertensão intracraniana, bulbar, medular, de insuficiência vertebrobasilar ${ }^{2,6,24,25}$.

Serviço de Neurologia e Neurocirurgia do Hospital Santa Isabel: *Neurocirurgião; **Neurologista. Aceite: 8-abril-1994.

Dr. José Alberto Gonçalves da Silva - Av. Minas Gerais 1150 - 58030-092 João Pessoa PB - Brasil. 
A primeira publicação sobre o tratamento cirúrgico da impressão basilar foi feita por Ebenius ${ }^{14}$, em 1934, tendo sido a primeira operação realizada em setembro de 1932, por Olivecrona. Dentre as malformações nervosas associadas à impressão basilar, a malformação de Arnold-Chiari, 81,9\% de nossa casuística, é a mais frequente $e^{2,7-10,13,16,18,21}$. Do ponto de vista histórico, a primeira descrição foi feita por Cleland ${ }^{12}$, em 1883, em estudos necroscópicos de 9 crianças, observando que o tronco cerebral era alongado e que o quarto ventrículo se estendia ao interior do canal cervical. Chiari ${ }^{11}$, em 1891 , observou que as amígdalas cerebelares se achavam localizadas no canal cervical e descreveu o caso de uma criança de 6 meses de idade, na qual a ponte, bulbo e quarto ventrículo cervical se achavam no interior do canal raqueano, alcançando a quinta vértebra cervical. Arnold ${ }^{3}$, em 1894, descreveu 1 caso de mielomeningocele lombossacra num prematuro ponderal, em que porções do cerebelo desciam em direção caudal, alcançando a porção média da coluna cervical.

A impressão basilar é anomalia muito frequente no Nordeste do Brasil, fato este verificado por diversos autores ${ }^{4,7-10,18-22}$. No entanto, as causas desta maior ocorrência permanecem desconhecidas. A partir de 1970 , passamos a ter maior interesse por esta patologia, em virtude de sua elevada ocorrência em nosso meio. A finalidade do presente estudo é apresentar os achados cirúrgicos observados em 260 casos operados em nosso Serviço.

\section{CASUÍSTICA E MÉTODO}

Nossa casuística consiste de 260 pacientes com malformações occipitocervicais, operados no período de março-1971 a outubro-1992. Destes, $29(11,1 \%)$ apresentavam impressão basilar pura; $18(6,9 \%)$, malformação de Arnold-Chiari; e 213 (81,9\%), impressão basilar associada à malformação de Arnold-Chiari. Quanto ao sexo, 156 (60\%) pacientes eram do sexo masculino e 104 (40\%) do feminino. A idade variou de 2 a 66 anos com média de 34,6 anos. Quanto ao tratamento neurocirúrgico empregado, os pacientes foram divididos em 2 grupos.

No grupo I estão os primeiros 64 pacientes, nos quais a operação consistiu em craniectomia occipital inferior com o paciente em posição sentada e moderada anteflexão da cabeça, laminectomia cervical alta, cuja extensão dependia do grau de migração das amígadalas cerebelares herniadas. A dura-máter permanecia aberta e suturada à musculatura cervical lateral. Neste grupo conseguiu-se, com especial cuidado cirúrgico, que a membrana aracnóide permanecesse intacta, na maior parte dos casos.

No grupo II estão os restantes 196 pacientes. Nestes, a dura-máter foi fechada com emprego de plástica. A maior parte dos pacientes deste grupo foi submetida a intubação endotraqueal sem retroflexão da cabeça e o posicionamento na mesa cirúrgica foi sentado, com a cabeça ereta ou em moderada anteflexão.

\section{RESULTADOS}

As anormalidades anatômicas encontradas durante a operação de nossos pacientes se acham nominadas na Tabela 1. As complicações pós-operatórias e a mortalidade são apresentadas nas Tabelas $2 \mathrm{e} 3$, respectivamente.

\section{COMENTÁRIOS}

Em relação ao tamanho da craniectomia deixamos de praticar a craniectomia restrita às imediações do forame magno, como proposta por vários autores ${ }^{8,9,13,15,24}$, e passamos a realizar craniectomia ampla da fossa posterior, baseados no fato de que a fossa posterior costuma ser menor na impressão basilar e na malformação de Arnold-Chiari, como foi demonstrado por Nyland ${ }^{29}$. Os cuidados com a intubação de paciente, a extubação ulterior, o posicionamento na mesa cirúrgica e a plástica da dura-máter representam fatores decisivos para o sucesso do ato cirúrgico.

Os limites de retroflexão da cabeça (para a intubação) e de anteflexão (para o posicionamento na mesa operatória) devem ser testados na fase pré-operatória. Os movimentos de ante e retroflexão da cabeça podem acarretar deslocamentos da apófise odontóide, anormalmente situada, comprimindo o bulbo e/ou medula cervical, desencadeando, desta forma, distúrbios respiratórios graves, lesões motoras e sensitivas. 
Tabela 1. Achados cirúrgicos.

\begin{tabular}{lcc}
\hline \multicolumn{1}{c}{ Tipo de achado } & $\mathrm{N}^{\circ}$ casos & $\%$ (260 casos) \\
\hline \hline Escama occipital papirácea & 77 & 29,6 \\
Escama occipital espessada & 39 & 15,0 \\
Ligamento atlantatoccipital espessado & 53 & 30,3 \\
Dura-máter sem pulsar & 38 & 14,6 \\
Aracnoidite & 175 & 67,3 \\
Bloqueio do forame de Magendie & 32 & 12,3 \\
Chiari I & 180 & 69,2 \\
Chiari II & 51 & 19,6 \\
Cisto amigdalar & 6 & 2,3 \\
Impressão cerebelar & 50 & 19,2 \\
Hemisfério cerebelar maior & 38 & 14,6 \\
Anomalias vasculares & 65 & 25,0 \\
Hidrocefalia & 13 & 5,0 \\
Siringomielia & 40 & 15,3 \\
\hline
\end{tabular}

Tabela 2. Impressão basilar elou Arnold-Chiari: causas de óbitos em 25 pacientes.

\begin{tabular}{lccc}
\hline & & $\%$ & $\%$ \\
\cline { 3 - 4 } \multicolumn{1}{c}{ Causas } & $N^{\circ}$ casos & 25 óbitos & 260 operados \\
\hline \hline Distúrbios respiratórios & 14 & 56 & 5,3 \\
Fístula e meningite & 5 & 20 & 1,9 \\
Hemorragia digestiva & 2 & 8 & 0,7 \\
Per-operatório & 1 & 4 & 0,3 \\
Crise hipertensiva + coma & 1 & 4 & 0,3 \\
Tetraplegia + coma & 1 & 4 & 0,3 \\
Hematoma subdural (válvula) & 1 & 4 & 0,3 \\
Total & 25 & 100 & 9,6 \\
\hline
\end{tabular}

Tabela 3. Mortalidade ( 260 casos de IB elou A-CH).

\begin{tabular}{cccccc}
\hline Grupo I (64 casos) & \multicolumn{3}{c}{ Grupo II (196 casos) } & Mortalidade global \\
$\mathrm{N}^{\circ}$ 6́bitos & $\%$ & $\mathrm{~N}^{\circ}$ óbitos & $\%$ & $\mathrm{~N}^{\circ}$ 6́bitos & $\%$ \\
12 & 18,7 & 13 & 6,6 & 25 & 9,6 \\
\hline
\end{tabular}

A extubação do paciente é outro fator de importância. Em nossa casuística, a extubação foi praticada durante vários anos, independentemente do tempo, mas baseada apenas no estado de lucidez apresentado pelas pacientes no pós-operatório. Entretanto, a partir de 1985, passamos a praticar a extubação no dia seguinte à operação, levando-se em conta a possibilidade de surgimento de distúrbios respiratórios, não só dependentes da própria patologia craniocervical mas, também, decorrentes de lesões pré-operatórias dos nervos glossofaríngeo e vago, de brevis collis e de macroglossia. 
A partir de 1975, passamos a utilizar plástica da dura-máter com as finalidades de aumentar o espaço anatômico da fossa posterior, evitar o aparecimento de fístula liquórica, impedir a penetração de secreção sanguinolenta na região operada (o que piora a aracnoidite), recompor a paquimeninge e permitir maior proteção às estruturas da fossa posterior. Em nossa experiência ${ }^{20}$, a plástica da duramáter contribuiu decisivamente para a diminuição do número de complicações pós-operatórias, especialmente dos distúrbios respiratórios e do índice de mortalidade.

A escama occipital e a região do forame magno são disformes em quase todos os pacientes. Em $77(29,6 \%)$ pacientes, a escama occipital era papirácea, tendo sua espessura, em vários casos, menos de $1 \mathrm{~mm}$, como foi verificado por Gatai ${ }^{17}$. Por outro lado, observamos, em 39 (15\%) pacientes, aumento anormal da espessura óssea, o que motivou em vários deles dificuldade técnica na realização da craniectomia.

A dura-máter comumente é mais espessada que o normal e, na região do forame magno, é mais profunda e deslocada no sentido cranial. O ligamento atlantoccipital bem como a dura-máter justaposta a este eram bastante espessados em 53 (20,3\%) pacientes. Espessamento e constricção da dura-máter foram verificados por diversos autores ${ }^{13,17}$. Em virtude do angustiamento da dura-máter e também do ligamento atlantoccipital, observamos a não pulsação da dura-máter em $38(14,6 \%)$ pacientes. A pulsação normal da fossa posterior foi verificada em alguns pacientes com a seç̧ão do ligamento atlantoccipital e em outros após a durotomia.

A aracnoidite da fossa posterior é achado realmente muito frequente e de grande importância na fisiopatogenia do quadro clínico da impressão basilar. Driesen ${ }^{13}$ admite que a aracnoidite é o fator mais importante para o desenvolvimento da sintomatologia neurológica. Em nossa casuística observamos a presença de aracnoidite em diferentes graus de intensidade em 175 (67,3\%) pacientes. Em decorrência da aracnoidite, as amígdalas cerebelares herniadas permanecem aderidas ao bulbo e medula cervical e, muitas vezes, estranguladas por aderências aracnóideas, adquirindo posição e conformação diferentes após a secção dos prolongamentos da membrana aracnóide.

$\mathrm{O}$ forame de Magendie pode ser obstruído pelo processo de aracnoidite, por membrana ou pela herniação das amígdalas cerebelares que comumente o cobrem. Em 32 (12,3\%) pacientes verificamos a obstrução total deste forame, especialmente nos casos associados a siringomielia, achado frequentemente registrado na literatura ${ }^{13,15,16}$.

A malformação de Arnold-Chiari é anomalia do rombencéfalo, constituída da migração caudal, isolada ou combinada de estruturas do cerebelo, ponte, bulbo e quarto ventrículo e, frequentemente, se acha associada à impressão basilar. Em nossa casuística de 260 casos de malformações occipitocervicais, verificamos a presença de $18(6,9 \%)$ casos de malformação de Arnold-Chiari isolada e $213(81,9 \%)$ casos de impressão basilar associada à malformação de Arnold-Chiari.

Dos 231 pacientes com malformação de Arnold-Chiari, $180(69,2 \%)$ eram do tipo I, isto é, apresentavam herniação apenas de estruturas cerebelares e $51(19,6 \%)$ do tipo II, nos quais havia deslocamento caudal do bulbo, parte da ponte e quarto ventrículo. $\mathrm{O}$ bulbo e a porção inicial da medula cervical eram disformes, em alguns casos apresentando-se o bulbo alongado e noutros encurtado e de forma arrendondada, achados também verificados por outros autores ${ }^{13,27,28}$.

As amígdalas cerebelares herniadas são comumente disformes, variando de delgadas a atróficas e de aumentadas de volume a bastante espessadas, bem como apresentavam topografia variável e coloração anêmica, esbranquiçada, amarelada e, por vezes, cor aparentemente normal. Quanto à topografia das amígdalas cerebelares, verificamos sua herniação bilateral e no mesmo nível em 143 $(61,9 \%)$ pacientes e níveis diferentes em 80 (34,6\%). A herniação unilateral foi detectada em 8 $(3,4 \%)$ pacientes.

Cisto, em uma amígdala cerebelar herniada, cujo conteúdo era claro e incolor, foi observado em $6(2,3 \%)$ casos. Achado semelhante foi verificado por List ${ }^{25}$ e Lougheed e col. ${ }^{26}$. Cisto na cisterna magna foi descrito por Garcin e Oeconomos ${ }^{15}$ e em um hemisfério cerebelar por Phillips ${ }^{30}$. 
Impressões provocadas no verme inferior e hemisférios cerebelares foram observadas em 50 $(19,2 \%)$ casos. Estas impressões decorrem da compressão da crista occipital interna e da elevação das bordas do forame magno sobre o cerebelo, fato também observado por Caetano de Barros ${ }^{7} \mathrm{e}$ Driesen $^{13}$. Em muitos casos, um hemisfério cerebelar é maior de que o outro e comumente apresenta conformação irregular, achado este verificado em $39(14,6 \%)$ casos.

As modificações da rede vascular da fossa posterior, observadas em casos de impressão basilar e malformação de Arnold-Chiari, provavelmente ocupam lugar de destaque na fisiopatologia do quadro neurológico bem como das complicações pós-operatórias ${ }^{20}$, pois podem acarretar distúrbios na suplência sanguínea de estruturas desta região. Entre as anomalias vasculares da fossa posterior, a mais frequentemente observada é a posição anormal da artéria cerebelar posteroinferior, descendo em sentido caudal e acompanhando ou não a herniação das amígdalas cerebelares ${ }^{7,9,13,18,24}$. Por vezes, o calibre de uma artéria poderá ser menor ou, até mesmo, uma ou ambas as artérias podem estar ausentes, como verificado por Driesen ${ }^{13}$. Em nossa casuística, durante a operação, verificamos a presença de $65(25,0 \%)$ casos de anomalias vasculares, em 30 uma ou ambas as artérias exibindo o "loop sign". Entre estes, 10 pacientes apresentaram agenesia de uma destas artérias, 6 agenesia bilateral; em 3 a artéria era hipoplásica e noutro ambas as artérias vertebrais apresentavam posição anômala, encobrindo parte da medula e bulbo. Em outros 23 casos, a malformação vascular se caracterizava por vasos pequenos, delgados e disformes, na maioria dos casos sobre as amígdalas herniadas, aparecendo também sobre o bulbo e porção inicial da medula espinhal.

Hidrocefalia é observada em alguns casos de impressão basilar. Seu mecanismo fisiopatológico poderá ser decorrente do fechamento dos forames de Magendie e Luschka por aracnoidite ou pela herniação das amígdalas cerebelares. Bloqueio total ou parcial do aqueduto de Sylvius e bloqueio cisternal também são descritos. Em nossa casuística 13 (5,0\%) casos apresentavam hidrocefalia.

Segundo Klaus ${ }^{24}$, Gustafson e Oldeberg, em 1940, publicaram pela primeira vez a ocorrência simultânea de impressão basilar e siringomielia, Ulteriormente, surgiram outras publicações a respeito do assunto ${ }^{8,9,15,30}$. Em nossa casuística, observamos a incidência de $15,3 \%$ de siringomielia em associação com a impressão basilar.

Quanto ao tratamento cirúrgico da siringomielia, empregamos nos 20 casos iniciais a descompressão da fossa posterior proposta por Gardner e Angel ${ }^{16}$, em 1958, acrescida, em alguns casos, de mielotomia mediana ou lateral ${ }^{22}$. Em virtude dos resultados não satisfatórios, praticamos nos 10 casos seguintes a ressecção das amígdalas cerebelares herniadas. Apesar de não termos observado qualquer complicação, deixamos de utilizar este método, pelos riscos inerentes de possível lesão da artéria cerebelar posterior e inferior que, em muitos casos, se encontra hipoplásica.

Bertrand ${ }^{5}$, em 1973, propôs a ressecção das amígdalas cerebelares herniadas com a finalidade de se obter melhores resultados no tratamento da siringomielia, enquanto Williams ${ }^{34}$, em 1978, intronduziu a aspiração intrapial da porção mediana de uma ou de ambas as amígdalas com fixação, à dura-máter lateral, da pia-máter que as envolve. Williams ${ }^{34,35}$ não emprega plástica da dura-máter. Fundamentados nos estudos de Williams, passamos nos últimos 10 casos de siringomielia a praticar a aspiração intrapial, não de parte, mas de toda a amígdala herniada bilateralmente, acrescida de fixação de sobra pial à dura-máter lateral, o que deixa o quarto ventrículo completamente aberto. Finalizamos a operação com plástica da dura-máter a fim de criar uma grande cisterna magna.

A utilização de fascia lata para a plástica dural apresenta como complicação o aparecimento de aderências com o cerebelo através de sua revascularização, o que dificulta em muito reoperações. Por este motivo, deixamos de utilizá-las. Passamos então a utilizar dura-máter de cadáver conservada em glicerina e, atualmente, empregamos pericárdio bovino (HP Bioengenharia, São Paulo) pois apresenta inúmeras vantagens em relação à dura-máter conservada em glicerina. Tanto a dura-máter conservada quanto o pericárdio bovino não são revascularizados e, por isso, não criam aderências ulteriores, como pudemos observar em alguns casos de siringomielia reoperados. 
Em todos os casos de siringomielia operados, retiramos o máximo de aracnóide da transição occipitocervical a fim de criar uma grande cisterna magna. Raftopoulos e col. ${ }^{31}$, por outro lado, empregam método de aspiração ultrassônica das amígdalas cerebelares com preservação do espaço subaracnóideo, tática mais importante do método.

Os pacientes, nos quais foi empregada a ressecção ou a aspiração intrapial das amígdalas cerebelares herniadas, vêm apresentando resultados promissores.

As complicações pós-operatórias que acarretaram óbito de pacientes de nossa série se encontram na Tabela 2. A causa mais frequente de óbito decorreu de distúrbios respiratórios, cuja instalação variou do $1^{\circ}$ ao $19^{\circ}$ dia de pós-operatório. Observaram-se outras complicações como fístula liquórica, meningite, hemorragia digestiva, óbito per-operatório, crise hipertensiva, tetraplegia e coma e hematoma subdural pós derivação ventriculoperitoneal para tratamento de hidrocefalia.

Os cuidados que, em fases diferentes, introduzimos no tratamento cirúrgico da impressão basilar, quanto à intubação e extubação do paciente, seu posicionamento na mesa cirúrgica, maior craniectomia e a plástica de dura-máter, definitivamente contribuíram na diminuição das complicações pós-operatórias e da mortalidade.

\section{REFERÊNCIAS}

1. Ackermann JF. Ueber die Kretinen, eine besondere Menschenabart in den Alpen. Gotha, in der Ettingerschen Buchhandlung, 1790.

2. Ackermann R, Wolff H. Neurologische Stoerungen bei Missbildungen am Schaedel-Halsuebergang (Basale Impression). Dt Z NervHeilk 1953, 170: 47-61.

3. Arnold J. Myelocyste, Transposition von Gewebskeimen und Sympodie. Beitr path Anat 1894, 16: 1-28.

4. Barbosa R. Impressão basilar (platibasia). J Bras Neurol 1955, 7: 247-266.

5. Bertrand G. Dynamic factors in the evolution of syringomyelia and syringobulbia. Clin Neurosurg 1973, 20: 322-333.

6. Brocher JEW. Die Occipito-Cervical-Gegend: eine diagnostish-pathogenetishe Studie. Stuttgart:Georg Thieme Verlag, 1955.

7. Caetano de Barros M. Contribuição ao estudo da impressão basilar associada à malformação de ArnoldChiari. Tese. Recife, Brasil, 1959.

8. Caetano de Barros M, Farias W, Ataíde L, Lins S. Basilar impression and Arnold-Chiari malformation: a study of 66 cases. J Neurol Neurosurg Psychiatry 1968, 31: 596-605.

9. Canelas HM, Zaclis J, Tenuto RA. Contribuição ao estudo das malformações occipitocervicais, particularmente da impressão basilar. Arq Neuropsiquiatr 1952, 10: 407-476.

10. Canelas HM, Tenuto RA, Zaclis J, Cruz OR. Malformações occipitocervicais: a propósito de vinte novos casos. Arq Neuropsiquiatr 1956, 14: 1-26.

11. Chiari H. Ueber Veraenderungen des Kleinhirns infolge von Hydrocephalie des Grosshirns. Dt med Wschr 1891, 17: 1172-1175.

12. Cleland J. Contribution to the study of spine bifida, encephalocele and anencephalus. J Anat Physiol 1883, 17: 257-292.

13. Driesen W. Operationsbefunde am Zentralnervensystem bei basilaren Impressionen und verwandten Missbildungen der atlanto-occipitalen Region. Acta Neurochir 1960, 9: 9-68.

14. Ebenius B. The roentgen appearance in four cases of basilar impression. Acta Radiol 1934, 15: 652-656.

15. Garcin $R$, Oeconomos $D$. Les aspects neurologiques des malformations congénitales de la charnière craniorachidienne. Paris: Masson, 1953.

16. Gardner WJ, Angel J. The mechanism of syringomyelia and its surgical correction. Clin Neurosurg 1958, 6: 131-140.

17. Gatai G. Ueber die chirurgische Behandlung der basalen Impression. Zbl Neurochir 1957, 17: 264-274.

18. Gonçalves da Silva JA. Resultados do tratamento cirúrgico da impressão basilar e malformação de ArnoldChiari: estudo de 72 casos. Tese. João Pessoa, 1977.

19. Gonçalves da Silva JA, Gonçalves da Silva CE, de Farias Brito JC, Rodrigues de Sousa JB. Impressão basilar e malformação de Arnold-Chiari: considerações técnico-cirúrgicas a propósito de 13 casos. Arq Neuropsiquiatr 1978, 36: 27-31. 
20. Gonçalves da Silva JA, Gonçalves da Silva CE. Postoperative Komplikationen bei 126 Faellen basilaerer Impression und Arnold-Chiarischer Missbildung. Neurochirurgia 1981, 24: 153-157.

21. Gonçalves da Silva JA, Cantisani Filho JU, Gonçalves da Silva CE. Malformação de Arnold-Chiari: análise de 6 casos operados. Arq Bras Neurocir 1982, 1: 173-186.

22. Gonçalves da Silva JA, Cantisani Filho JU, de Farias Brito JC, da Nóbrega PV, Viana NO, da Silva EB, Gonçalves da Silva CE. Impressão basilar, Arnold-Chiari e siringomielia: análise de 20 casos operados. Arq Bras Neurocir 1987, 6: 77-95.

23. Homén EA. Zur Kenntniss der rhachitischen (?) Deformationen der Schaedelbasis und der basalen Schaedelhyperostosen. Dtsch Z NervHeilk 1901, 20: 3-15.

24. Klaus E. Die basilaere Impression. Leipzig: S Hirzel, 1969.

25. List CF. Neurologic syndromes accompanying developmental anomalies of occipital bone, atlas and axis. Arch Neurol Psychiatry 1941, 45: 577-616.

26. Lougheed LE. Arnold-Chiari malformation in adults: report of case. Proc Staff Meet Mayo Clin 1957, 32: 632-636.

27. McConnell AA, Parker HL. A deformity of the hind-brain associated with internal hydrocephalus: its relation to the Arnold-Chiari malformation. Brain 1938, 61: 415-429.

28. Mohr PD, Strang FA, Sambrook MA, Boddie HG. The clinical and surgical features in 40 patients with primary cerebellar ectopia (adult Chiari malformation). Quart J Med 1977, 181: 85-96.

29. Nyland H, Krogness KG. Size of posterior fossa in Chiari type 1 malformation in adults. Acta Neurochir 1978, 40: 233-242.

30. Phillips DG. Basilar impression. J Neurol Neurosurg Psychiatry 1955, 18: 58-67.

31. Raftopoulos C, Sanchez A, Matos C, Balériaux D, Bank WO, Brotchi J. Hydrosyringomyelia-Chiari I complex: prospective evaluation of a modified foramen magnum decompression procedure. Preliminary results.

Surg Neurol 1993, 39: 163-169.

32. Virchow R. Untersuchungen ueber die Entwicklung des Schaedelgrundes im gesunden und krankhaften Zustande und ueber den Einfluss derselben auf Schaedelform, Gesichtsbildung und Gehirnbau. Berlin: Georg Reimer, 1857.

33. Virchow R. Beitraege zur physischen Anthropologie der Deutschen, mit besonderer Beruecksichtigung der Friesen. Berlin: Buchdruckerei der koeniglichen Akademie der Wissenschaften, 1876.

34. Williams B. A critical appraisal of posterior fossa surgery for communicating syringomyelia. Brain 1978, 101: 223-250.

35. Williams B. Surgical treatment of syringobulbia. Surg Brain Stem 1993, 4: 533-571. 